\title{
Excitations of domain walls pinned at F/AF interface steps
}

Article in IEEE Transactions on Magnetics · October 2000

DOI: 10.1109/20.908676 · Source: IEEE Xplore

CITATION

1

2 authors:

\section{Ana L. Dantas}

Universidade do Estado do Rio Grande do Nort.. 53 PUBLICATIONS 285 CITATIONS

SEE PROFILE
READS

5

Some of the authors of this publication are also working on these related projects: 


\title{
Excitations of Domain Walls Pinned at F/AF Interface Steps
}

\author{
Ana L. Dantas and Artur S. Carriço
}

\begin{abstract}
We study rigid displacement excitations of a Nèel wall of a thin uniaxial ferromagnetic film on a two-sublattice antiferromagnetic substrate. An interface step defect pins the domain wall and provides the restoring force that stabilizes the domain wall oscillations. The frequency of the rigid displacement domain wall modes is shown to be of the same order of magnitude as that of the uniform domain modes of the ferromagnetic film, for a wide range of interface exchange energy values.
\end{abstract}

Index Terms-Domain wall excitations, domain walls, interface defect.

\section{INTRODUCTION}

$\mathbf{T}$ HE magnetic properties of thin ferromagnetic $(F)$ films grown on antiferromagnetic (AF) substrates may be controlled to a large extent by the nature of the interface. Interface roughness in F/AF bilayers is a subject of current interest and despite the research effort dedicated to the subject and the large amount of experimental data accumulated so far, there are still unsolved questions [1], [2].

The value of the exchange field coupling the magnetic moments across the interface is a key parameter for the interface exchange bias field which controls the shift of the hysteresis loops. It has been shown that AF islands at the interface, exposing different sublattices of the AF, may nucleate walls, either in the $\mathrm{AF}$ or $\mathrm{F}$, and the energy of these walls give the correct order of magnitude of the interface biasing field [3].

While it seems that in low Miller indices F/AF interfaces, field cooled from the Nèel temperature of the substrate, the interface magnetic pattern is still a subject of discussion [1], a satisfactory control of the interface magnetic structure has been achieved in $\mathrm{Fe} / \mathrm{Cr} / \mathrm{Fe}$ trilayers [4]. In this study [4] the formation of a periodic stripe domain structure in thin Fe films was discussed. This structure was reported to be in good correspondence with the $\mathrm{Cr}$ magnetic structure in the terraces formed in the $\mathrm{Fe} / \mathrm{Cr}$ interface. The magnetic pattern of the $\mathrm{Fe}$ film indicates that the interface consists of uniform alternating regions, each of which having a single value of the effective interface field acting on the magnetic moments of the thin Fe film. The magnetic properties of this kind of interface might help to learn about low Miller indices rough interfaces.

Manuscript received September 14, 2000. This work was supported in part by the CNPq

A. L. Dantas is with the Departamento de Física, Universidade do Estado do Rio Grande do Norte, 59600-900, Mossoró - RN, Brazil (e-mail analunat@zaz.com.br).

A. S. Carriço is with the Departamento de Física Teórica a Experimental, Universidade Federal do Rio Grande do Norte, 59072-970, Natal - RN, Brazil (e-mail: acarrico@dfte.ufrn.br).

Publisher Item Identifier S 0018-9464(00)08825-7.

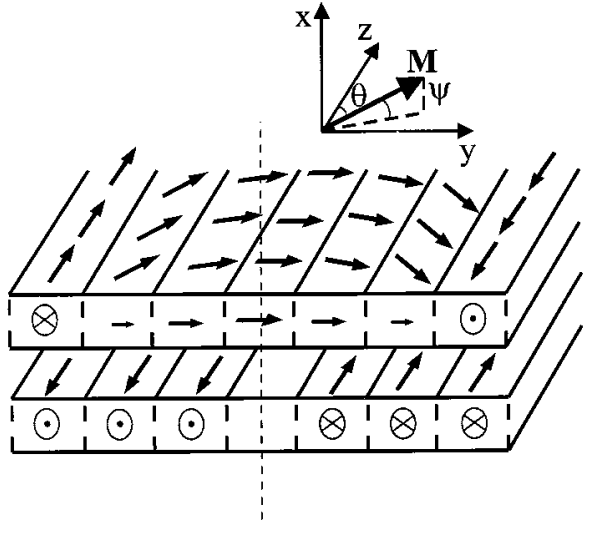

Fig. 1. Schematic representation of a Nèel wall pinned at a F/AF interface step defect.

A common feature of the magnetic structure of thin $\mathrm{Fe}$ films on vicinal $\mathrm{Cr}$ substrates [4] and that of $\mathrm{Fe}$ films on two-sublattices AF substrates is the existence of interface areas with opposite sublattices of the substrate. The border of the interface regions where there is a change of the substrate sublattice act, in both cases, as domain wall nucleation centers. The equilibrium properties of these domain walls [5], as well as the domain wall excitations, can be explored to probe the interface exchange coupling in a local manner. The details of the excitation spectrum of a domain wall in a single defect, as appropriate to small angle vicinal $\mathrm{Cr}$ substrates, with terraces much wider than the Fe domain wall width, might be helpful to study the effect of interface roughness in F/AF bilayers.

In this contribution we discuss the pinning of a domain wall at an interface defect in a F/AF system. We consider a Nèel wall of a thin uniaxial ferromagnetic film on a two-sublattice AF substrate, as shown in Fig. 1. The uniaxial direction of the substrate is supposed to coincide with the easy axis direction ( $z$-axis) of the ferromagnet. The normal to the surface is along the $x$-axis, and a step, running along the $z$-direction, divides the substrate into two regions, each containing spins from a single sublattice of the antiferromagnet. In Fig. 1 only the interface AF plane is shown. The AF film consists of a stacking of atomic layers each of which has moments from a single sublattice. The magnetic arrangement consists of atomic planes where each other repeats a given sublattice. This is the case, for instance, of a $\mathrm{FeF}_{2}(010)$ substrate or that of a $\mathrm{NiO}(111)$ substrate. Therefore a step defect in the interface, comprising an odd number of planes, leads to the magnetic pattern shown schematically in the picture.

Domain wall nucleation is favored by the change in the interface exchange energy at the step defect. The domain wall state has an energy lower than that of the uniform state, along the 
$z$-axis, if the interface exchange energy overcomes the domain wall energy $(4 \sqrt{A K})$. In the case of large domains this condition is met even for small values of the interface exchange energy constant $(J)$.

For small values $(J)$ one expects to have a magnetization profile controlled by the intrinsic exchange and anisotropy energies of the ferromagnet. In this case although the interface energy is responsible for the domain wall nucleation and stability, the domain wall structure itself is not affected. However, if $(J)$ is large, a considerable reduction of the domain wall width may be produced. In this case any property which originates from the magnetic profile within the domain wall region is associated to a rather small fraction of the sample. We show below that the restoring force which stabilizes rigid displacement domain wall oscillations are due to energy fluctuations within the domain wall. Thus the frequency of these excitations might help to study the microscopic details of the interface magnetic structure.

\section{Equilibrium StRUCtURE OF THE DOMAIN WALL}

The magnetic energy per unit wall area is given by:

$$
\begin{aligned}
E=\int_{-L}^{L}\left\{A\left(\frac{d \theta}{d y}\right)^{2}-K \cos ^{2} \theta-(J(y)+H M) \cos \theta\right. \\
\left.+2 \pi M^{2} \sin ^{2} \psi \sin ^{2} \theta\right\} d y
\end{aligned}
$$

where

$A$ is the exchange stiffness constant,

$K$ is the uniaxial anisotropy constant,

$J(y)$ is the interface exchange energy,

$H \quad$ is the external field applied along the $z$-axis,

$M \quad$ is the magnetization and

$\theta \quad$ is the angle of the magnetization with the $z$-axis.

The last term in (1) represents the magnetostatic energy of the surface magnetic charge density produced by the small out-ofplane component of the magnetization, controlled by the angle $\psi$. The change of substrate sublattice at $y=0$ is represented by a step discontinuity of $J(y)$ at $y=0$. We take $J(y)=J$ for $y<0$ and $J(y)=-J$ for $y>0$. The anisotropy field is $H_{A}=2 \mathrm{~K} / \mathrm{M}$ and the interface field, on each domain, is $H_{J}=J / M$. The interface and field effects are incorporated in the magnetic profile given by:

$$
\tan \left(\frac{\theta_{0}}{2}\right)=\exp \left(\frac{y-q_{H}}{\Delta}\right)
$$

For the equilibrium profile we have $\psi=0$ and the values of the displacement of the wall from the pining center, $q_{H}$, and the domain wall width, $\Delta$, are found by minimizing the energy. $q_{H}$ and $\Delta$ are given by:

$$
q=\Delta \operatorname{arcth}\left(\frac{H M}{J}\right)
$$

and

$$
\Delta=\frac{\Delta_{0}}{\sqrt{1+\frac{J}{K} \ln 2}}
$$

where $\Delta_{0}=\sqrt{A / K}$.

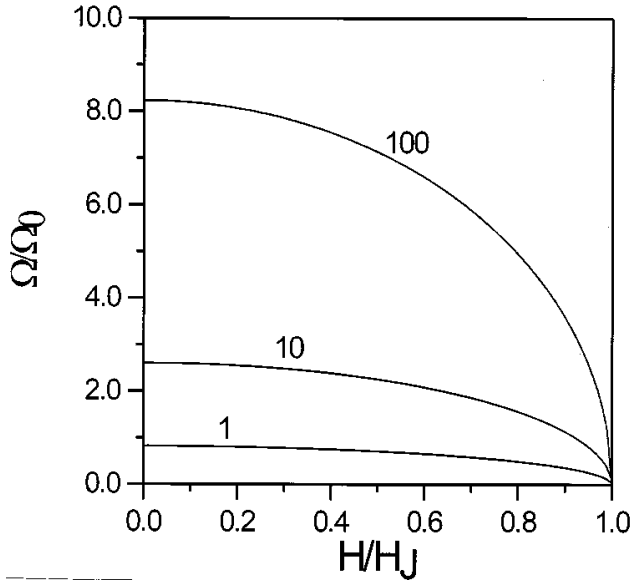

Fig. 2. Rigid displacement domain wall excitations. The frequency is scaled by the frequency of the uniform mode of the domains. The number by the curves indicate the values of $J / K$. See text for further details.

Equations (3) and (4) indicate that the domain wall center is located at the step defect $\left(q_{H}=0\right)$, in the absence of external field, and also that for an external field strength of $H=J / M$ the domain wall is liberated from the step defect. Since the domain wall displacement increases too rapidly for large values of $H$, in (4) we have neglected the field effects. Notice that, as predicted earlier [5], a large reduction of the domain wall width occur if the interface exchange energy is much larger than the anisotropy energy.

\section{Rigid DisPlaCEMENT EXCITATIONS}

The frequency of rigid displacement domain wall oscillations are found by integrating the Landau-Lifshitz equations in the domain wall [6]. We use $\theta(y, t)=\theta_{0}(y-\eta(t))$ where $\theta_{0}(y)$ is given by (2) and $\eta(t)=\eta e^{i w t}$. We also consider a small, oscillating, out-of-plane angle $\psi(t)=\psi^{i w t}$ which produces the demagnetizing energy term in (1).

The equations of motion are given by:

$$
\frac{d \eta}{d t}=2 \frac{\gamma}{M} \frac{\partial E}{\partial \psi}
$$

and

$$
\frac{d \psi}{d t}=-2 \frac{\gamma}{M} \frac{\partial E}{\partial \eta} .
$$

Notice from (1) that $\partial E / \partial \psi$ is proportional to an integral of $\sin ^{2} \theta$, since we are assuming small values of the amplitude of the out of plane angle. $\partial E / \partial \psi$ is also proportional to an integral of $\sin ^{2} \theta$. Thus the frequency of the rigid displacement oscillations are determined by the magnetic structure within the domain wall region.

The frequency of the domain wall oscillations is given by:

$$
\frac{\Omega}{\Omega_{0}}=\sqrt{\frac{4 \pi M H_{J}}{H_{A}\left(H_{A}+4 \pi M\right) \cosh ^{2}\left(\tanh ^{-1}\left(H / H_{J}\right)\right)}} .
$$

As seen in Fig. 2 the frequency of domain wall oscillations is a monotonically decreasing function of $H$ and $\Omega=0$ when $H=H_{J}$. The curves correspond to $H_{J} / H_{A}=1,10$ and 100 . We used $H_{A}=0.55 \mathrm{kOe}$ as appropriate to Fe thin films. As seen in Fig. 2, the frequency is scaled by $\Omega=\gamma \sqrt{H_{A}\left(H_{A}+4 \pi M\right)}$ 
and the domain wall oscillation frequency falls in the same range of the uniform oscillations in the domains.

\section{CONCLUSION}

We remark that the scale of the frequency axis in Fig. 2 is $\Omega_{0}=\gamma \sqrt{H_{A}\left(H_{A}+4 \pi M\right)}$. Notice that even when $J / 2 K$ changes by two orders of magnitude the maximum value of the frequency of the domain wall excitation remains of the same order of magnitude of $\Omega_{0}$. This is of interest for the study of roughness in F/AF interfaces. As indicated by the shift of the hysteresis curves commonly measured, the interface exchange field would be of the order of the anisotropy of the ferromagnet. However in hysteresis measurements one may be sampling the average effect of the interface field, which may be larger than the measured hysteresis shift by two to three orders of magnitude. Thus the weak dependence of the spectrum of domain wall excitations on the strength of the interface field is a valuable feature. It should be possible to observe interface pinned domain wall modes in experimental set ups designed for FMR measurements.

The weak dependence of the domain wall spectrum in the strength of the interface field results from the fact that when $J$ increases there is a reduction of the domain wall width. Thus, although the density of interface energy increases, the overall effect on the domain wall is not as strong.

The monotonic decrease of $\Omega(H)$ might also be helpful to identify the domain wall response in resonance experiments with the external field parallel to one of the domains.

The study of domain wall excitations has been applied in the past for a number of magnetic systems. See, for instance, [7]-[11] and references therein, for works on the properties of bubble materials, as well as general features of domain wall excitations and domain wall mobility.

The excitation of domain wall modes has been, more recently, used to study domain wall profiles in Co (0001) thin films with stripe domains. In this work ferromagnetic resonance techniques have been explored to selectively probe the excitations of particular regions of the stripe domain structure [12].

In this contribution we have shown that the rigid displacement excitations of a domain wall pinned by an interface step defect in a F/AF bilayer may help to estimate the strength of the interface exchange interaction, since the restoring force which stabilize the oscillations are due to an energy balance within the domain wall region. Therefore the measurement of the domain wall excitations frequency inform about the interface exchange coupling in microscopic areas of the interface.

The reduction of the domain wall width by the interface field reported earlier [5] is confirmed in this work. From (4) it is easily seen that for low anisotropy materials the domain wall width of an interface pinned wall may be significantly smaller than the intrinsic value $\left(\Delta_{0}\right)$. Thus, in the case of strong interface coupling, the excitation frequency results from an average over an even smaller area of the interface. This might be a promising means of accessing the local value of the interface exchange field for F/AF bilayers made of low anisotropy ferromagnets.

A similar situation is found in the study of the inter-film coupling in trilayers, such as $\mathrm{Fe} / \mathrm{Cu} / \mathrm{Fe}$. In these systems fluctuations in the spacer thickness may lead to considerable changes in the effective coupling. It has been recently shown [13] that the measurement of domain wall resonance in magnetic trilayers might be a promising means to probe the local value of the inter-film exchange coupling. The restoring force of an optical mode of domain wall pairs was shown to be proportional to the inter-film coupling energy integrated within the domain wall.

\section{REFERENCES}

[1] J. Nogués and I. K. Schuller, "Exchange Bias," J. Magn. Magn. Mat., vol. 192, pp. 203-233, 1999.

[2] D. T. Pierce, J. Unguris, R. J. Celotta, and M. D. Stiles, "Effect of roughness, frustration, and antiferromagnetic order on magnetic coupling of Fe/Cr multilayers," J. Magn. Magn. Mat., vol. 200, pp. 290-321, 1999.

[3] A. P. Malozemoff, "Random-field model of exchange anisotropy at rough ferromagnetic-antiferromagnetic interfaces," Phys. Rev. B, vol. 35, pp. 3679-3682, 1987.

[4] J. Unguris, R. J. Celotta, and D. T. Pierce, "Observation of two different oscillation periods in the exchange coupling of $\mathrm{Fe} / \mathrm{Cr} / \mathrm{Fe}(100)$," Phys. Rev. Lett., vol. 67, pp. 140-143, 1991.

[5] A. L. Dantas and A. S. Carriço, "Domain wall pinning at an interface step defect," J. Phys.: Cond. Matter, vol. 11, pp. 2707-2717, 1999.

[6] A. H. Morrish, The Physical Principles of Magnetism. New York, NY, USA: J. Willey, 1965.

[7] J. C. Slonczewski, "Dynamics of domains and domain walls in soft magnetic-films," IEEE Trans. Magn., vol. 27, pp. 3532-3538, 1991.

[8] - "Dynamics of magnetic domains and walls," J. Appl. Phys., vol. 69 , pp. 4590-4592, 1991

[9] - "Breathing vibrations of a Nèel wall," J. Appl. Phys., vol. 55, pp. 2536-2538, 1984

[10] B. E. Argyle and J. C. Slonczewski, "Continuous-wave domain-wall oscillations in bubble garnet-films," J. Appl. Phys., vol. 52, pp. 2353-2353, 1981.

[11] A. P. Malozemoff and J. C. Slonczewski, Magnetic Domain Walls in Bubble Materials. New York, NY: Academic Press, 1979.

[12] U. Ebels, P. E. Wigen, and K. Ounadjela, "Probing domain wall structures in $\mathrm{Co}(0001)$ thin films using ferromagnetic resonance," Europhys. Lett., vol. 46, pp. 94-100, 1999.

[13] R. L. Stamps, A. S. Carriço, and P. E. Wigen, "Domain-wall resonance in exchange-coupled magnetic films," Phys. Rev. B, vol. 55, pp. 6473-6484, 1997. 